\title{
BEYOND SLOW-FAST: RELAXATION OSCILLATIONS IN SINGULARLY PERTURBED NONSMOOTH SYSTEMS
}

\author{
SAMUEL JELBART
}

(Received 8 May 2021; first published online 10 June 2021)

2020 Mathematics subject classification: primary 37C75; secondary 34A34, 34D15, 34E15, 37C10, $37 \mathrm{C} 27$.

Keywords and phrases: dynamical system, ordinary differential equation, geometric singular perturbation theory, nonsmooth system, oscillation, desingularisation.

Sharp dynamical transitions are ubiquitous in nature and can be found in the dynamics of fluid flow near an airfoil or in mechanical oscillations resulting from the competing forces of static and dynamic friction. Frequently, dynamical transitions are modelled mathematically by inserting a nonsmooth interface, leading to a piecewise smooth (PWS) system. For applications requiring detailed understanding of the dynamics associated with the transition itself, however, nonsmooth approximations of this kind may not be valid, and singularly perturbed models which limit to PWS systems as some $\epsilon \rightarrow 0$ must be adopted. The analytical difficulties associated with smooth transitions in singular perturbation problems are notorious, although significant progress has been made for an important subclass, slow-fast problems, where a combination of geometric singular perturbation theory (GSPT) and a method for geometric desingularisation known as blow-up have proven especially useful. This thesis constitutes part of a research project which aims to address the question: 'to what extent can GSPT and blow-up methods be combined with PWS theory in order to study the dynamics of transitions more generally, that is, beyond the scope of slow-fast problems?' We focus on a small number of case studies arising primarily in the study of mechanical and electrical oscillators, paying particular attention to the occurrence of relaxation oscillations that are not necessarily slow-fast, but nonetheless consist of qualitatively distinct dynamical phases in a nonsmooth singular limit $\epsilon \rightarrow 0$.

First, we are able to show that a particular class of relaxation oscillations of this kind can be approximated by systems with corresponding slow-fast systems, which we analyse using a coordinate-independent GSPT that is able to cope with nontrivial timescale separations inherent in these systems (see [4]). Our analysis demonstrates the

Thesis submitted to the University of Sydney in July 2020; degree approved on 15 October 2020; principal supervisor Martin Wechselberger and co-supervisor Kristian Uldall Kristiansen.

(C) 2021 Australian Mathematical Publishing Association Inc. 
power and simplicity of such an approach. Motivated by our findings, we investigate a relationship between PWS systems, GSPT and systems with exponential nonlinearities via a detailed analysis of two electrical oscillators which may be considered as 'prototypes' for a second class of (not necessarily slow-fast) relaxation oscillations, which cannot in general be analysed via corresponding slow-fast systems in the same manner as the first class (see [1]). This analysis is complicated by the presence of certain essential singularities, which pose significant difficulties for blow-up methods. By utilising recent advances in blow-up techniques, we are able to derive detailed asymptotic information and prove existence for the observed relaxation oscillations. Finally, we study the onset of (non-slow-fast) relaxation oscillations in systems undergoing a PWS bifurcation known as the boundary-focus bifurcation in the singular limit $\epsilon \rightarrow 0$. We show that the PWS dynamics is sufficient to qualitatively determine the bifurcation structure associated with the smooth transitions for $0<\epsilon \ll 1$, but insufficient to describe the more subtle dynamical changes associated with the transition from regular to relaxation-type oscillations, which must be obtained using methods from GSPT and blow-up. Some of this research has been published in [2, 3].

Throughout the thesis, emphasis is placed on the methods and techniques being applied, which we believe to be applicable quite generally for the study of abrupt dynamical transitions.

\title{
References
}

[1] S. Jelbart, K. U. Kristiansen, P. Szmolyan and M. Wechselberger, 'Singularly perturbed oscillators with exponential nonlinearities', Preprint, 2019, arXiv:1912.11769.

[2] S. Jelbart, K. U. Kristiansen and M. Wechselberger, 'Singularly perturbed boundary-focus bifurcations', Preprint, 2020, arXiv:2006.06087.

[3] S. Jelbart, K. U. Kristiansen and M. Wechselberger, 'Singularly perturbed boundary-equilibrium bifurcations', Preprint, 2021, arXiv:2103.09613.

[4] S. Jelbart and M. Wechselberger, 'Two-stroke relaxation oscillators', Nonlinearity 33(5) (2020), Article ID 2364.

\author{
SAMUEL JELBART, School of Mathematics and Statistics, \\ University of Sydney, Camperdown, \\ New South Wales 2006, Australia \\ e-mail: jelbart@ma.tum.de
}

